\title{
Fibrin Glue Injection for Cavernous Sinus Hemostasis Associated with Cranial Nerve Deficit: A Case Report
}

\author{
Daryoush Tavanaiepour $^{1}$ Sarah Jernigan ${ }^{2}$ Mohamad Abolfotoh ${ }^{3}$ Ossama Al-Mefty ${ }^{4}$
}

${ }^{1}$ Department of Neurosurgery, University of Florida College of Medicine, Jacksonville, Florida, United States

2 Department of Neurosurgery, University of Miami, Miami, Florida, United States

${ }^{3}$ Department of Neurosurgery, Ain Shams University, Cairo, Egypt

${ }^{4}$ Department of Neurosurgery, Brigham and Women's Hospital and Harvard School of Medicine, Boston, Massachusetts, United States

Address for correspondence Daryoush Tavanaiepour, MD, Department of Neurosurgery, Tower 1, 8th floor, 580 West 8th Street, Jacksonville, FL 32209 (e-mail: Daryoush.tavanaiepour@jax.ufl.edu).

J Neurol Surg Rep 2015;76:e72-e74.

\begin{abstract}
Fibrin glue injection has been used to control intraoperative cavernous sinus (CS) venous bleeding. There have been no reported complications related to this maneuver. We present a case where a patient developed a sensory trigeminal nerve deficit after injection of fibrin glue into the posterior CS during resection of a petrosal meningioma. We believe that this deficit was due to the compression of the trigeminal ganglion

Keywords

- fibrin glue

- cavernous sinus

- cranial nerve similar to balloon compression procedures. Although fibrin glue injection may achieve satisfactory cavernous sinus homeostasis, the volume and rate of injection should be kept in mind to avoid a compressive lesion on traversing cranial nerves and surrounding structures, or retrograde filling of the venous tributaries.
\end{abstract}

\section{Introduction}

Fibrin glue injection (Baxter Healthcare Corp., Deerfield, Illinois, United States) has been used to control intraoperative cavernous sinus (CS) venous bleeding. There have been no reported complications related to this maneuver. ${ }^{1,2}$ We present a case in which a patient developed a sensory trigeminal nerve (TN) deficit after injection of fibrin glue into the posterior CS during resection of a petrosal meningioma. We believe this deficit was due to the compression of the trigeminal ganglion (TG) similar to balloon compression procedures but persistent until the glue resolves.

\section{Case Report}

A 40-year-old woman with radiation-induced petrous apex meningioma underwent resection of the lesion via a posterior fossa approach. After complete resection of the lesion, the involved dura was excised to the junction of the superior

received

October 10, 2014

accepted

November 10, 2014

published online

March 4, 2015 petrosal sinus and the posterior wall of the CS, where venous hemorrhage ensued. This was easily controlled with injection of fibrin glue into the posterior CS. The remaining of the procedure was uneventful. Postoperatively the patient developed new-onset ipsilateral facial numbness. There was dense hypoalgesia and hypoesthesia in the trigeminal V1 and V2 distributions, and less affected, in V3. The motor trigeminal function was intact. Postoperative magnetic resonance imaging (MRI) demonstrated total gross resection; however, there was expansion of the CS by the fibrin glue. Repeated postoperative MRI at 3 months demonstrated resolution of the CS expansion (-Fig. 1). However, the patient's sensory TN deficit persisted after the 18-month follow-up.

\section{Discussion}

Multiple intraoperative techniques have been described for cavernous sinus hemostasis that include the use of Surgicel
License terms

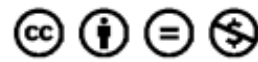

(c) 2015 Georg Thieme Verlag KG
Stuttgart · New York $10.1055 / \mathrm{s}-0034-1543977$ ISSN 2193-6366. 


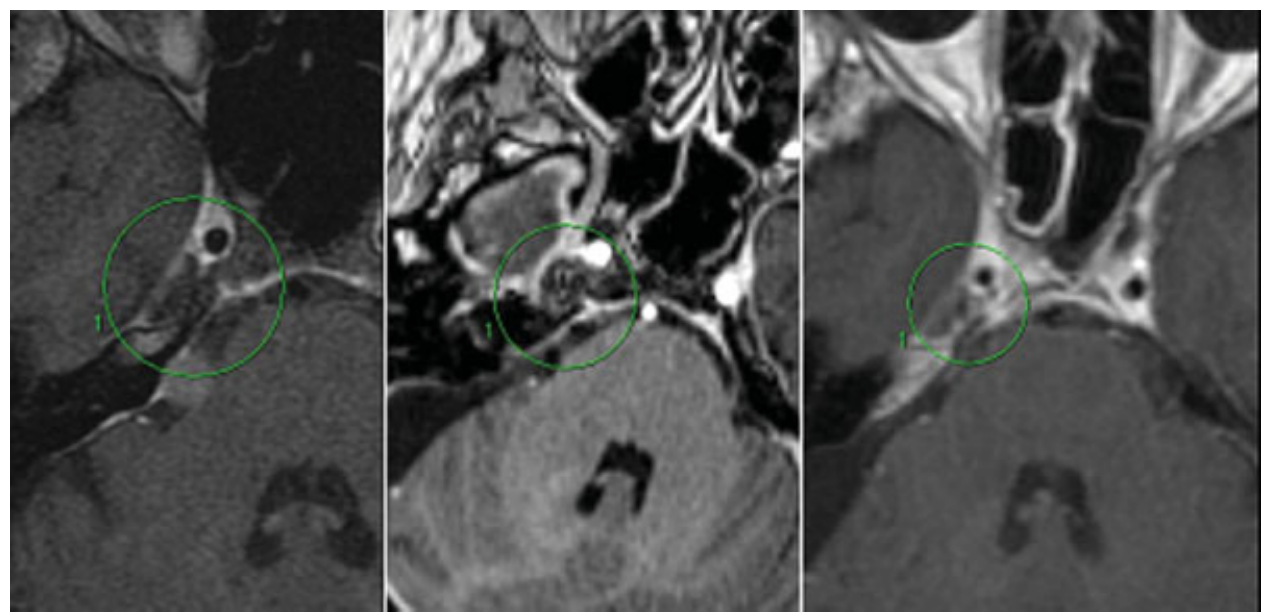

Fig. 1 Preoperative magnetic resonance imaging (MRI) demonstrating part of the lesion with normal configuration of the cavernous sinus (left panel). Postoperative MRI demonstrating nonenhancing expansion of the cavernous sinus due to fibrin glue injection (center panel). Repeat postoperative MRI at 3 months demonstrating resolution of the expansion (right panel).

(Ethicon Inc., Somerville, New Jersey, United States), thrombin-soaked Gelfoam (Pfizer, New York, New York, United States), FloSeal (Baxter Biosciences, Vienna, Austria), and fibrin glue. ${ }^{3-5}$ Although each technique has varying degrees of hemostasis, specific complications associated with these maneuvers have not been documented. Fibrin glue injection into the CS has been used safely and beneficially to control venous bleeding, ${ }^{1,2}$ but controlling the pressure and the amount may be crucial in avoiding complications. To our knowledge, this is the first case report describing a cranial nerve deficit following fibrin glue injection into the CS. The CS expansion on the postoperative MRI is attributed to the fibrin glue that produced a compressive lesion on the trigeminal ganglion with the resultant sensory deficit.

This situation is analogous to percutaneous balloon compression (PBC) of the TG for the treatment of trigeminal neuralgia. The development of $\mathrm{PBC}$ for trigeminal neuralgia originated from successful reports of surgical manipulation of the TN for trigeminal neuralgia. Shelden et al performed proximal root compression at the TG that involved applying "gentle" pressure to the nerve using a blunt dissector with complete resolution of symptoms and with no sensory deficit. $^{6}$ This procedure was associated with a recurrence rate of $\sim$ $20 \%{ }^{3}$ Later, Mullan and Lichtor introduced the PBC procedure for trigeminal neuralgia. ${ }^{7}$

Sensory complaints associated with PBC have been well documented. In a large retrospective case series, 441 of 496 patients undergoing PBC had early facial numbness (89\%). However, the facial numbness persisted for $>3$ months in only 23 patients $(4.6 \%){ }^{8}$ Despite its prevalent use, there has been a wide variation of the $\mathrm{PBC}$ parameters including the amount of pressure $(459-2080 \mathrm{~mm} \mathrm{Hg})$, duration (0.5-15 minutes), and volume $(0.5-1.1 \mathrm{~mL})$ of balloon compression. ${ }^{9}$ Studies have demonstrated a direct correlation between the duration of balloon compression and the development of facial numbness. Lee and Chen performed PBC in 80 patients, while keeping the other parameters stable, the duration of compression was either 60 seconds or 180 seconds. At 1-year follow-up, patients with a shorter duration of compression had lower rates of facial numbness, with similar rates of efficacy. ${ }^{9}$ Brown and Pilitsis also demonstrated an association between duration of $\mathrm{PBC}$ compression (1.18 versus 1.06 minutes) and facial numbness in 65 PBC procedures. ${ }^{10}$ Zanusso et al performed $\mathrm{PBC}$ with intraoperative pressure monitoring and demonstrated an association between high PBC pressure with greater side effects but with lower recurrence rates at 1 year. ${ }^{11}$ Similarly, Brown and Pilitsis demonstrated a trend toward facial numbness with higher $\mathrm{PBC}$ pressures. $^{10}$

Drawing analogies from the preceding discussion regarding $\mathrm{PBC}$ and TN sensory deficits, the fibrin glue injection produced a compressive lesion on the TG, similar to PBC, leading to the patient's facial numbness. In this case, the duration of TG compression was sustained for days (evident by the postoperative MRI), as compared with seconds for PBC. The amount of pressure applied to the TG by the fibrin glue injection is unknown, although it was sufficient to produce the sensory deficit without a motor deficit.

\section{Conclusion}

Although fibrin glue injection may achieve satisfactory cavernous sinus homeostasis, the volume and rate of injection should be kept in mind to avoid a compressive lesion on traversing cranial nerves and surrounding structures or retrograde filling of the venous tributaries.

\section{References}

1 Krayenbühl N, Hafez A, Hernesniemi JA, Krisht AF. Taming the cavernous sinus: technique of hemostasis using fibrin glue. Neurosurgery 2007;61(3, Suppl):E52,;discussion E52

2 Sekhar LN, Natarajan SK, Manning T, Bhagawati D. The use of fibrin glue to stop venous bleeding in the epidural space, vertebral venous plexus, and anterior cavernous sinus: technical note. Neurosurgery 2007;61(3, Suppl):E51; discussion E51 
e74 Fibrin Glue and Cavernous Sinus Hemostasis Tavanaiepour et al.

3 Phookan G, Davis AT, Holmes B. Hemangioendothelioma of the cavernous sinus: case report. Neurosurgery 1998;42(5): 1153-1155; discussion 1155-1156

4 Fraser JF, Mass AY, Brown S, Anand VK, Schwartz TH. Transnasal endoscopic resection of a cavernous sinus hemangioma: technical note and review of the literature. Skull Base 2008;18(5):309-315

5 Bedi AD, Toms SA, Dehdashti AR. Use of hemostatic matrix for hemostasis of the cavernous sinus during endoscopic endonasal pituitary and suprasellar tumor surgery. Skull Base 2011;21(3): 189-192

6 Shelden CH, Pudenz RH, Freshwater DB, Crue BL. Compression rather than decompression for trigeminal neuralgia. J Neurosurg 1955;12(2):123-126

7 Mullan S, Lichtor T. Percutaneous microcompression of the trigeminal ganglion for trigeminal neuralgia. J Neurosurg 1983;59(6): 1007-1012
8 Skirving DJ, Dan NG. A 20-year review of percutaneous balloon compression of the trigeminal ganglion. J Neurosurg 2001;94(6): 913-917

9 Lee ST, Chen JF. Percutaneous trigeminal ganglion balloon compression for treatment of trigeminal neuralgia, part II: results related to compression duration. Surg Neurol 2003;60(2): 149-153; discussion 153-154

10 Brown JA, Pilitsis JG. Percutaneous balloon compression for the treatment of trigeminal neuralgia: results in 56 patients based on balloon compression pressure monitoring. Neurosurg Focus 2005; 18(5):E10

11 Zanusso M, Curri D, Landi A, Colombo F, Volpin L, Cervellini P. Pressure monitoring inside Meckel's cave during percutaneous microcompression of gasserian ganglion. Stereotact Funct Neurosurg 1991;56(1):37-43 